\title{
Repeptization and the Theory of Electrocratic Colloids
}

\author{
G. FRENS ${ }^{1}$ AND J. TH. G. OVERBEEK
}

van't Hoffaboratorium der Rijksuniversiteit Sterrenbos 19 , Utrecht, Netherlands.

Received September 9, 1970; Accepted March 17, 1971

\begin{abstract}
The coagulation and the repeptization of electrocratic colloids can be treated in one theory provided that the appropriate boundary conditions are chosen. From this version of the DLVO theory it follows that for each sol there exists a critical value $Z_{\infty}{ }^{c}$ of the double layer parameter $Z_{\infty}, Z_{\infty}=2 e \varphi_{\delta} / k T$. A sol is stable, and flocs can repeptize if $Z_{\infty}>Z_{\infty} c$. For $Z_{\infty}<Z_{\infty} c$ the traditional DLVO theory is obtained. Some examples are given to illustrate this concept. In the appendix the interaction of double layers with a constant charge is related to that at a constant potential.
\end{abstract}

\section{INTRODUCTION}

Repeptization experiments, where the flocs from an electrocratic sol are redispersed by washing away the coagulating salt, seem to have no ready explanation in a theory for electrocratic colloids which considers double layer repulsion and van der Waals attraction as the only sources of interaction between colloidal particles. This has led to speculations about "third forces" and "impenetrable barriers" between the particles of repeptizable colloids.

Nearly all electrocratic colloids (e.g., $\mathrm{AgI}(1,2), \mathrm{BaSO}_{4}$ (3), oxides, sulfides (4), latices (5), even metal sols (6-9) show repeptization phenomena. Therefore the cause of repeptizability has to be rather nonspecific and of a general nature.

The effects of the counterion valency on the repeptizability of a floc $(1,4,10)$ point to double layer repulsion as the force which can break up the flocs in an electrocratic colloid. In this paper we shall show that it is not necessary to assume other forces than double layer replusion and van der Waals attraction in order to explain repeptization. To this end an adapted version of the DLVO theory will be developed, which accounts

\footnotetext{
1 Present address: Philips Research Laboratories, Findhoven, Netherlands.
}

for the experimental observations on repeptization, without losing its value for the explanation of the coagulation and the stability of electrocratic sols.

\section{EXPERIMENTAL OBSERVATIONS}

A review of experimental observations on coagulation and repeptization is beyond the purpose of this paper. For coagulation data we refer to textbooks. For repeptization we have extracted a number of general statements from the available literature. Experimental details and procedures are found in the original papers.

a) Repeptization of electrocratic colloids is possible $(1-4,8)$. It is not always complete, and depends on the electrolyte which was used to form the flocs.

b) Washing away the coagulant is often enough to cause repeptization $(4,8)$.

c) The addition of potential-determining ("peptizing") ions to the washing liquid helps repeptization $(2,4)$.

d) Fresh flocs are easier to repeptize than aged aggregates $(1,4,8)$. The rate at which the repeptizability of flocs is lost differs greatly among different colloidal materials. The permanent repeptizability of latices and clay suspensions is an exception.

e) The rate at which repeptizability of a 
floc disappears is the faster, the higher the temperature (2).

f) The influence of co-ions on repeptization is not large (4). It is specific for the combination of ion and colloid. There is no strong correlation with the valency of the co-ions.

g) Repeptization is relatively easy when a floc contains monovalent counterions. Polyvalent counterions (and also strongly adsorbed counterions) in a floc hinder its redispersion $(1,4,8,11)$.

h) Polyvalent counterions in a floc can be exchanged for monovalent ions, which makes such flocs repeptizable $(10,15)$.

j) The presence of bulky adsorbed ions or molecules, such as citrate, phosphate, EDTA, or acetone, in a sol can produce repeptizability $(12,13)$.

k) Repeptization depends on the concentration of the washing solution. It is the ionic character of the solute which is important, and not the osmotic value of this solution $(1,14)$.

I) Repeptization can be a very fast process $(1,8)$.

\section{THEORETICAL}

Coagulation and repeptization are both rate processes (16). In every rate process there are two conditions to be fulfilled:

1) The total free energy of the system decreases in the course of the process. This condition determines the direction in which there can be a rate process.

2) The individual events which constitute the process must not be improbable: the activation energy for such an event (i.c., for the collision of two particles so that they can form an aggregate) must not be too high. This second condition governs the rate of a process, but only for processes which are possible under the first condition.

According to the first of these two conditions the potential energy $V_{i}{ }^{0}$ of two aggregated particles (measured relative to a system of two particles which are too far apart to interact) must be negative in a coagulating colloid, and positive under repeptization conditions.

Repeptization is an experimental fact. Therefore the traditional position that $V_{t}^{0}$ is always negative because of the strong attraction between particles at short distances is untenable. This apparent contradiction is caused by the boundary conditions which are introduced in the theory to describe a nearly coagulating colloid. A theory for coagulating sols (high salt concentration, moderate and large distances) as well as for repeptizing flocs (low concentration, small interparticle distance) can be obtained when three modifications are introduced in the customary framework of the stability theory.

1) There is a distance of closest approach for two electrocratic particles. Not only is it impossible that the surface atoms of the particles interpenetrate (Born repulsion), but it is also highly improbable that a Brownian collision brings two particles closer together than twice the distance $\delta$ between a particle surface and the Outer Helmholtz Plane of the electrical double layer.

2) The double layer repulsion of two particles at a distance $H>2 \delta$ depends on the Stern potential $\varphi_{\delta}$, and $\varphi_{\delta}$ is a function of the electrolyte concentration.

3) During a Brownian collision it is the charge $\sigma$ and not the potential of the double layer system which remains constant.

\section{CONSTANT CHARGE VERSUS CONSTANT POTENTIAL}

Neither the forcing together of crossed Pt-wires (21) or $\mathrm{Hg}$ drops (22) which are kept at a well-defined potential nor the gradual thinning of a soap film (23) is perfect as an analog of the Brownian collision between two colloidal particles. These model experiments are very much unlike a Brownian collision in their time scale. This reflects the traditional assumption that a colloidal particle remains in equilibrium with its surroundings during a collision. Under this assumption the time scale of the collision does not enter the picture. 
We have shown earlier (24) that the assumption of electrochemical equilibrium at the particle-solution interface during a Brownian collision of two colloidal particles is unrealistic, even for so-called nonpolarizable interfaces. A Brownian collision takes about $10^{-7}$ sec, but a time of seconds is needed before a discharging current has restored the equilibrium in the disturbed double layers. Therefore it is the charge $\sigma$ and not the potential $\varphi$ of the double layer which remains constant during the collision.

In a discussion of flocs and their behavior we deal with interparticle distances that are much shorter than the Debye length $1 / \kappa$, and then the repulsion for constant charge is rather different from that at constant potential.

In the appendix the potential energy of the double layer repulsion at constant $\sigma$ $\left(V_{R}{ }^{\sigma}\right)$ is calculated. For flat plates we find:

$$
\begin{aligned}
V_{R}{ }^{\sigma}=V_{R}{ }^{\psi} & +\frac{8 n k T}{\kappa}\left[\left(Z_{H}-Z_{\infty}\right) \sinh \frac{Z_{\infty}}{2}\right. \\
& \left.-2\left(\cosh \frac{Z_{H}}{2}-\cosh \frac{Z_{\infty}}{2}\right)\right] \cdot \quad
\end{aligned}
$$

After writing $f(\mathrm{U}, \mathrm{z})=z^{2} \kappa^{-1} V_{R}$ (for flat plates) we obtain the repulsion energy of two spherical double layers from Eq. [1] by Derjaguin's approximation (27). The result is:

$$
\begin{aligned}
V_{R}{ }^{\sigma}= & \frac{2 \pi a}{z^{2}}\left[\int_{H_{0}}^{\infty} f_{(\mathrm{U}, \mathrm{Z})}^{\varphi} \mathrm{d} \frac{\kappa H_{0}}{2}\right. \\
& \left.+\int_{H_{0}}^{\infty}\left(f_{(\mathrm{U}, \mathrm{Z})}^{\sigma}-f_{(\mathrm{U}, \mathrm{Z})}^{\varphi}\right) \mathrm{d} \frac{\kappa H_{0}}{2}\right] .
\end{aligned}
$$

The first term in [2] is $V_{R}^{\varphi}$ as it is found in the constant potential version of the DLVO theory for spherical double layers. The second term is the correction which results from the assumption that $\sigma$ remains constant during the collision. Figure 1 illustrates that according to Eq. [2] the interaction curve for constant $\sigma$ is steeper than for constant $\varphi$, especially at low double layer potentials. The differences between $V_{R}{ }^{\sigma}$ and $V_{R}{ }^{\varphi}$ become small for $\kappa H_{0}>1$.

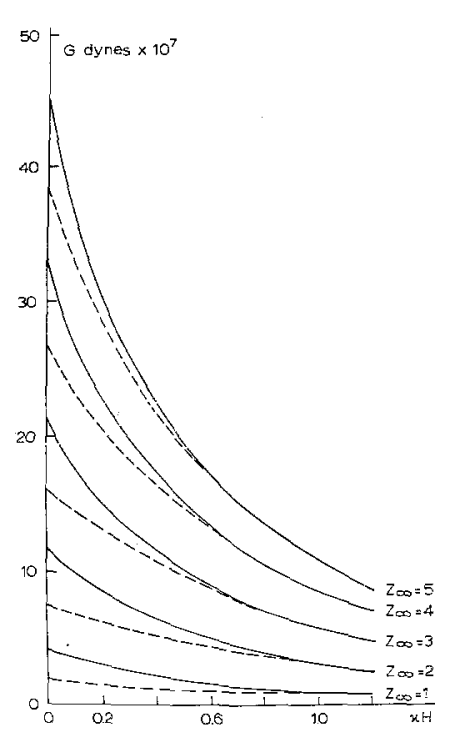

FIG. 1. Repulsion between spherical double layers, $G=z^{2} a^{-1} V_{R}$ Drawn: constant $\sigma$ dotted: constant $\varphi_{\delta}$.

\section{DISTANCE OF CLOSEST APPROACH}

Hamaker (28) calculated the potential energy $V_{A}$ due to the van der Waals attraction between two particles. In the nonretarded case, and in an approximation for small interparticle distance

$$
V_{A}=-A / 12 \pi H^{2}
$$

for flat plates, and

$$
V_{A}=-a A / 12 H
$$

for spherical particles. Here $A$ is the "Hamaker constant", and $H$ is the distance between the centers of the surface atoms of the particles.

The surfaces of two particles (of the same material) coincide when $I I$ equals the diameter $\Delta$ of the atoms in the particles. For two van de Waals crystals one obtains an estimate of the Hamaker constant $A$ by equating

$$
V_{A}=-A / 12 \pi \Delta^{2}=-2 \gamma,
$$

where $\gamma$ is the surface free energy of the particles and $\Delta$ is of the order of $3 \AA$.

If, however, the double layer charge remains constant in a Brownian collision it is 
impossible for two electrocratic particles to come as close as $H=\Delta$ during such a collision. Between the two surfaces there has to remain room for the counterions which maintain the electroneutrality of the double layer systems.

The distance of closest approach for two electrocratic particles is therefore $H=2 \delta$, where $\delta$ is the distance between the center of the surface atoms of a particle and the Outer Helmboltz Plane in the double layer at the surface. ${ }^{2}$

A reasonable estimate for this distance of closest approach is $4 \AA<H=2 \delta<10 \AA$.

For $H<2 \delta$ counterions are squeezed from between the particle surfaces. Such a violation of the electroneutrality of the double layer systems would give rise to a strong repulsion, which cannot be overcome by the relatively weak van der Waals attraction between the particles. This is nicely illustrated by the floc structure of clays at high salt concentrations. In these suspensions the platelets have a constant charge which remains fixed in the lattice. In the coagula the platelets are stacked face to face with layers of counterions between them (12).

Even for spherical particles, where the free counterions could be pushed aside in a lateral direction over distances of the order $1 / \kappa$, it can be seen that the distance of closest approach will not fall much below $2 \delta$ as long as $\kappa a$ is large. Only when the particles begin to lose their charge by (slow) desorp-

${ }^{2}$ Recently Levine (25) criticized our approach by pointing out that at a constant charge $\varphi_{\delta} \rightarrow \infty$ for $H \rightarrow 2 \delta$. He concludes that therefore $\sigma$ cannot remain constant. His criticism is valid, but his conclusion is false. There is indeed a steep rise of $\varphi_{\delta}$, but in Fig. 5 we find that this is for $\kappa H \ll 0.1$ where the O. H. P.'s are small fractions of an $\AA$ apart. The true consequence of this is that our assumption of a distance of closest approach $H=2 \delta$ is not rigorously exact: particles will not come closer than the repulsion permits them to come. It is, however, a very good approximation, better than our knowledge about the value of $\delta$ itself.

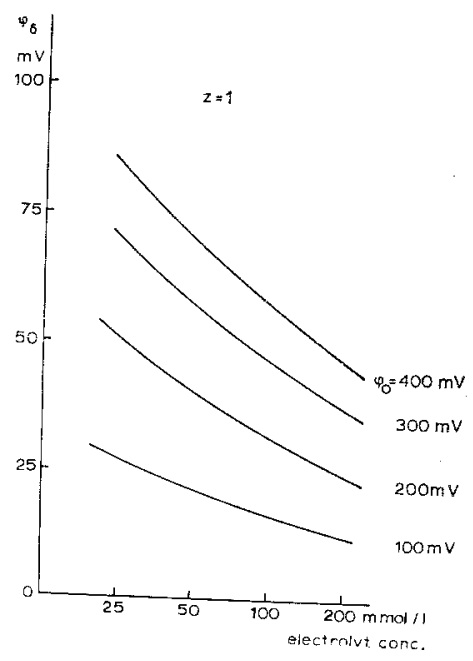

FIG. 2. Relation between $\varphi^{0}, \varphi_{\delta}$ and $C$ for $z=1$, according to (30).

tion reactions does it become possible for the counterions to get out of the way.

\section{THE STERN POTENTIAL}

The Stern potential $\varphi_{\delta}$ of a particle in a $0.1 M$ electrolyte solution differs from $\varphi_{\delta}$ at a concentration of $0.001 M$. This has nothing to do with the question whether $\sigma$ or $\varphi$ is constant during a Brownian collision. It is a direct consequence of the Stern-Grahame description of the electrical double layer and its capacity (29). Using the Gouy Chapman. theory and a constant capacity of 15 $\mu \mathrm{F} / \mathrm{cm}^{2}$ for the Stern layer Reerink obtained (30) Fig. 2. It is borne out that $\varphi_{\delta}$ is quite sensitive to changes in $C$, and rather insensitive to variations in $\varphi^{\circ}$ in the range of $C$ and $\varphi^{0}$ which is of interest in colloid chemistry.

This same conclusion is reached from the much more refined double layer models that have been worked out by Levine (31). In aqueous suspension $\varphi_{\delta}$ varies between 0 and $100 \mathrm{mV}$ as a function of the (not too low) salt concentration.

Many authors equate $\varphi_{i}=\zeta$, and use the electrokinetic $\zeta$-potential as the basis for their DLVO calculations. Such values of $\zeta$ give indeed an idea of the magnitude of the potential difference $\varphi_{\delta}$ across the diffuse 
layer, and they illustrate again that $\varphi_{\delta}$ depends on the electrolyte concentration.

The fact that $\varphi_{\delta}$ is certainly much lower than $\varphi^{0}$ introduces a difference from some of the older versions of the DLVO theory. Reasonings based on the assumption that the double layer potentials are high lose their validity when $\varphi_{\delta}$ is used instead of $\varphi^{0}$.

\section{POTENTIAL ENERGY DIAGRAMS}

Rate processes like coagulation and repeptization are conveniently discussed by means of potential energy diagrams $\left(V_{t}=\right.$ $V_{R}+V_{A}$ as a function of $\left.H\right)$. The experimental properties of the system enter into the construction of such a diagram through the parameters $Z_{\infty}, \kappa, \delta$, the Hamaker constant $A$, and the particle radius $a$.

We consider spherical particles. $V_{R}$ is given by Eq. [2] with $H_{0}=H-2 \delta, V_{A}$ is calculated with the simple Hamaker formula (4) for small $H / a$. The more elaborate versions of the theory of $V_{R}$ and $V_{A}(28,31)$ would change details in the results, but the approximate version which is used here serves well to illustrate the effect of the three alternative boundary conditions on the DLVO theory. For a start we shall choose typical values for $Z_{\infty}, \kappa, \delta, A$, and $a$. Later on we shall discuss the effect of variations in these parameters on the potential energy diagram. For $a$ we have chosen $a=500 \AA$, somewhere in the middle range of colloidal dimensions. A distance of closest approach $2 \delta=4 \AA$ leaves room for a small counterion near the point of contact between the particles. A Hamaker constant $A=5 \times 10^{-13}$ erg seems a reasonable estimate for particles in the hydrosols of AgI and other, nonconducting, materials (35).

For coagulation conditions we take a salt concentration of 150 mmoles/1 1-1 electrolyte (which is the "coagulation concentration" $C^{c}$ for the AgI hydrosol (2)). The Stern potential $\varphi_{\delta}=25 \mathrm{mV}$, the kind of value that is obtained by equating $\zeta=\varphi_{\delta}$ for a coagulating sol $(33,34)$, and which is supported by the model calculations (30).

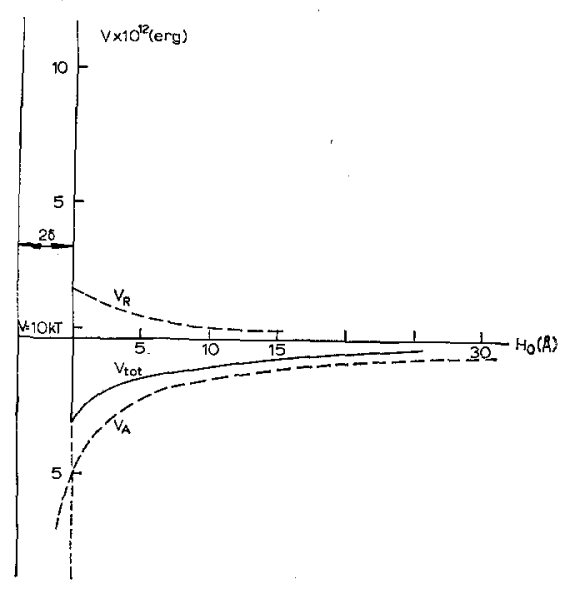

Fig. 3. Potential energy diagram for coagulation conditions. $\mathrm{A}=5 \times 10^{-13} \mathrm{erg}, a=5 \times 10^{-6}$ $\mathrm{cm}, \delta=2 \times 10^{-8} \mathrm{~cm}, \mathrm{C}=150 \mathrm{mmol} \mathrm{l}^{-1}, z=1$, $Z_{\infty}=1$.

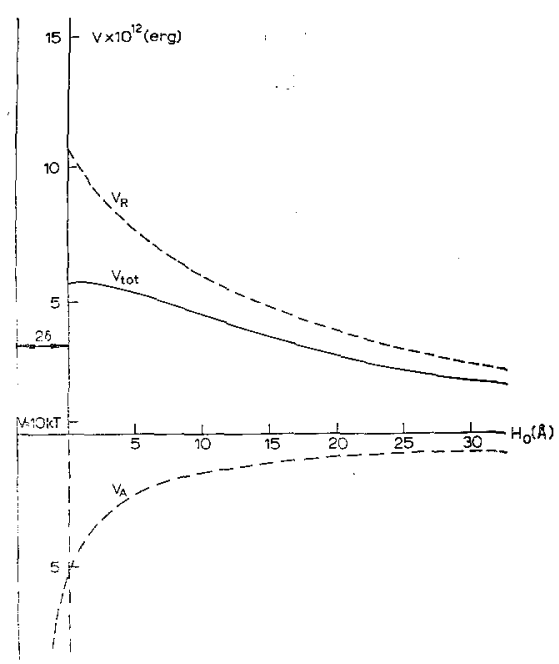

Fig. 4. Potential energy diagram for repeptization conditions and in stable sols. $A=5 \times 10^{-13}$ erg, $a=5 \times 10^{-6} \mathrm{~cm}, \delta=2 \times 10^{-8} \mathrm{~cm}, \mathrm{C}=15$ $\mathrm{mmol} \mathrm{1} 1^{-1}, z=1, Z_{\infty}=3$.

For a stable sol or for a repeptizing system the salt concentration is $10 \times$ lower ( 15 mmoles/1 1-1 electrolyte) and $\varphi_{8}$ is $75 \mathrm{mV}$. The value $\varphi_{\delta}=75 \mathrm{mV}$ is again a reasonable potential for a stable electrocratic colloid as follows from Fig. 2 ( $c f$. reference 32 ). With these parameters Figs. 3 and 4 are obtained. Indeed Fig. 3 predicts coagulation, and Fig. 
4 represents a stable sol or repeptizing flocs. The important feature of Figs. 3 and 4 is the difference in the potential energy $V_{i}{ }^{0}$ of an aggregate. It is negative in Fig. 3 and since no energy barrier exists there will be rapid coagulation. $V_{t}^{0}$ is positive in Fig. 4. Aggregates which might exist will repeptize-and rapidly, since again there is not much of an activation energy.

With $V_{t}^{0}>0$ a Brownian collision of free particles cannot result in their sticking together as a permanent aggregate. Such a sol is thermodynamically stable against coagulation. It may coarsen through "Ostwald ripening," but not by aggregation of the particles.

Neither Fig. 3 nor Fig. 4 is of the familiar ("diuturnal" (36)) type of potential energy diagram, where there is a maximum at moderate, and a deep minimum at very small interparticle distances. Such a diagram is obtained when $d V_{t} / d H$ is positive for small $H$, i.e. when $-V_{A}$ is a steeper function of $H$ than $V_{R}$. Diuturnal-type potential energy diagrams would be obtained if it were assumed that $A$ is very large, or that particles can come closer than atomic dimensions would let them $(H<$ $2 \delta$ ). Neither case is physically realistic. The only circumstances where diuturnal-type diagrams can be obtained in practice are those of a low charge in the diffuse layer combined with a low concentration of the intermicellar electrolyte (37).

\section{VARIATION OF PARAMETERS}

Variations in the parameters $\kappa, a, A, \delta$, and $Z_{\infty}$ can affect the rate processes of coagulation and repeptization by changing $V_{t}^{0}$ from negative to positive, or by affecting the shape of the potential energy diagram, and thus the rate of coagulation or repeptization. For $a \gg \delta$, which is the normal case, $V_{R}$ and $V_{A}$ are both proportional to the particle radius $a$. The shape of the $V_{t}-H$ diagram is therefore independent of $a$, and the choice of $a$ has no influence on whether $V_{t}^{0}$ is positive or negative.
The energy scale of the diagram is proportional to $a$, and the ratio $V_{i} / k T$ increases and decreases with the particle radius. This ratio enters into Fuchs' theory for the rate of the process. When the colloidal particles are crystallites with planes and edges and if a small activation energy remains it is favorable that aggregates are formed with such orientations of the particles that the small radii of curvature come together. In such systems the rate of slow coagulation will therefore not strongly depend on the average particle size, and the flocs will be rather loose structures. By the same token small particles and loose flocs have high rates of repeptization if there is an activation energy for that process at all.

The electrolyte concentration $C$ of a sol affects the parameters $Z_{\infty}$ (through the variation of $\varphi_{j}$ ) and $\kappa$. Of these two the variation in $Z_{\infty}$ is the more important (cf. Fig. 1), $V_{t}^{0}$ is independent of $\kappa$, but changes in $\kappa$ give changes in $d V_{t} / d H$. In the particular cases where $V_{t}^{0}<0$ and a "diuturnal" colloid is on the verge of coagulation this can still have a considerable effect on the coagulation rate. This is demonstrated in the classical version of the DLVO theory, where $a, A, Z_{\infty}$, and $\delta$ are chosen so that $V_{t}^{0}<0$ and then kept constant under variations of $\kappa$.

It is the essential point of a theory for coagulation as well as for repeptization, that $V_{t}^{0}$ can have both negative and positive values. The change in $V_{t}^{0}$ from negative to positive is, for a given colloid, caused by the change in $Z_{\infty}$.

Let us introduce the symbol $Z_{\infty}{ }^{c}$ for the critical value of $Z_{\infty}$, where $V_{t}^{0}=0$. Each colloid has a $Z_{\infty}{ }^{c}$, and $Z_{\infty}{ }^{c}$ is independent of $a$ and of $\kappa$. For $Z_{\infty}>Z_{\infty}{ }^{\circ}$ a sol is stable and aggregates tend to repeptization; for $Z_{\infty}<$ $Z_{\infty}{ }^{c}$ there can only be coagulation if there is any rate process at all.

The parameters $A$ and $\delta$ describe the different colloidal materials, and $Z_{\infty}^{c}$ relates these to the strength $\left(Z_{\infty}\right)$ of the double layer that can protect a given sol from 
TABLE I

Combination of the Hamaker Constant a and the Distance of Closest Approach $2 \delta$ Which MAKe $V_{R}+V_{A}=0$ AT $H=2 \delta(1-1$ ELECTROLYTE)

\begin{tabular}{|c|c|c|c|}
\hline$A\left(\right.$ ergs $\left.\times 10^{12}\right)$ & $Z_{\infty}{ }^{c}=1$ & 2 & 3 \\
\hline & $2 \delta(\AA)$ & $2 \delta(\AA)$ & $2 \delta(\hat{A})$ \\
\hline 0.2 & 0.4 & 0.1 & 0.1 \\
\hline 0.5 & 1 & 0.4 & 0.2 \\
\hline 1 & 2 & 0.7 & 0.4 \\
\hline 2 & 4 & 1.4 & 0.8 \\
\hline 3 & 6 & 2.1 & 1.2 \\
\hline 4 & 8 & 2.8 & 1.5 \\
\hline 5 & 10 & 3.5 & 1.9 \\
\hline 6 & 12 & 4.2 & 2.3 \\
\hline 7 & 14 & 5.0 & 2.7 \\
\hline 8 & 16 & 5.7 & 3.1 \\
\hline 9 & 18 & 6.4 & 3.5 \\
\hline 10 & 20 & 7.1 & 3.9 \\
\hline
\end{tabular}

coagulation. This is explored in Table I. In this table $A$ and $Z_{\infty}{ }^{c}$ are varied independently, and this generates the corresponding values of $2 \delta$. The variation of $A$ and $Z_{\infty}{ }^{c}$ is chosen between the limits $10^{-14}<A \leqq$ $10^{-12}$ erg and $1 \leqq Z_{\infty}{ }^{c} \leqq 3(1-1$ electrolyte $)$ to cover a wide range of practical situations. The lower limit for the distance of closet approach is about $H=2 \delta=4 \AA$. There is no upper limit for $2 \delta$ when bulky ions are chemisorbed but in the absence of specific adsorption $4<2 \delta<10 \AA$ seems reasonable.

From the data in Table I two types of conclusions can be drawn:

1) The requirement for a complete theory that $V_{t}^{0}$ can be positive as well as negative is met by many combinations of plausible numerical values for the parameters $Z_{\infty}{ }^{c}, \delta$, and $A$, and therefore the concept of a $Z_{\infty}{ }^{\circ}$ can be applied in many colloid chemical situations.

2) In most colloids $10^{-13}<A<10^{-12}$ erg, and for most counterions $4<2 \delta<$ $10 \AA$. The theory predicts that for these systems, and for $1-1$ electrolytes $1<Z_{\infty}{ }^{\circ}$ $<2$, i.e., $25<\varphi_{\delta}^{c}<50 \mathrm{mV}$. For colloids with a small $A$ (polymer latices) the value of $Z_{\infty}^{c}$ is also very small. It is the introduction of the parameter $Z_{\infty}{ }^{c}$ for each given colloid, and the notion that the variation of $Z_{\infty}$ relative to $Z_{\infty}{ }^{\circ}$ can influence the rate processes in colloids, which constitute the most important result of our version of the stability theory. This may be illustrated by the following examples.

a. Sols with a Low Double Layer Charge. A sol is stable for $Z_{\infty}>Z_{\infty}{ }^{\circ}$, and there can be coagulation if $Z_{\infty}<Z_{\infty}{ }^{c}$. The value $\varphi_{\delta}^{c}$ bears some resemblance to the critical $\zeta$-potential $\zeta^{c}$ of earlier experimenters. However, $\varphi_{\delta}{ }^{c} \neq \zeta^{c}$ even if it is assumed that $\varphi_{\delta}=\zeta$. A sol can still be stable for $\varphi_{\delta}<$ $\varphi_{\delta}^{c}$ in the sense that its rate of coagulation is negligible. If $\varphi_{\delta}<\varphi_{\delta}^{c}$ the rate of coagulation can still be small if the electrolyte concentration is also very small (37)

If the charge $\sigma$ of the double layer is so small that $Z_{\infty}<Z_{\infty}{ }^{\circ}$ even for $\kappa \rightarrow 0$ we have a "diuturnal" sol. It is stable at low salt concentrations, but its flocs cannot be redispersed by washing away the electrolyte.

b. Polyvalent Counterions in Repeptization Experiments. Both $Z_{\infty}\left(=z e \varphi_{\delta} / k T\right)$ and $V_{R}^{0}$ at $H_{0}=0\left(=a z^{-2} G^{0}\right)$ depend on the valency $z$. Let us assume that a given sol has $Z_{\infty}{ }^{c}=2$ at $z=1$. Then we obtain $V_{R}{ }^{0}$ at $Z_{\infty}{ }^{c}$ for $z=1$ from Eq. [2]. By definition this $V_{R}{ }^{0}=-V_{A}{ }^{0} \cdot V_{A}{ }^{0}$ is due to van der Waals attraction, and therefore independent of the ion valency $z$. Thus $V_{R}{ }^{0}$ at $Z_{\infty}{ }^{c}$ is also independent of $z$, and $G^{0}$ at $Z_{\infty}{ }^{c}$ (Fig. 1) is proportional to $z^{2}$.

Calculation shows that if

$$
\begin{array}{ll}
Z_{\infty}{ }^{c}=2 & \text { for } z=1, \text { then } \\
Z_{\infty}{ }^{c}=5.2 & \text { for } z=2, \text { and } \\
Z_{\infty}{ }^{c}=9 & \text { for } z=3 .
\end{array}
$$

This corresponds to $\varphi_{\delta}^{c}=50,65$, and 75 $\mathrm{mV}$ for uni-, di- and trivalent counterions, respectively.

Here is a reason why repeptization is so difficult for flocs with polyvalent counterions. In their presence $\varphi_{\delta}^{c}$ is high. In order that there be repeptization it is necessary that $\varphi_{\delta}$ be even higher $\left(Z_{\infty}>Z_{\infty}{ }^{c}\right)$. However, the strong adsorption of polyvalent ions 
even at fairly low concentrations tends to lower $\varphi_{\hat{\delta}}$, thus effectively preventing repeptization.

Conditions become more favorable for repeptization when the polyvalent ions are exchanged for monovalent counterions (10, 15). This is what can be observed in practice, e.g., when the $\mathrm{Ca}^{2+}$ ions which cause the open floc structure of arable clay soils are exchanged for $\mathrm{Na}^{+}$during a flooding with seawater. Under the salt water the flocs remain intact. Rain after the sea has withdrawn dilutes the coagulating electrolyte. Since the counterions are now univalent $Z_{\infty}{ }^{c}$ is lower after the exchange than it was before the flooding. $Z_{\infty}$ can rise above $Z_{\infty}{ }^{c}$. At $Z_{\infty}>$ $Z_{\infty}{ }^{c}$ the soil repeptizes and settles as a dense sediment-unfit for agriculture. Treatment of the flooded land with $\mathrm{Ca}^{2+}$-salts keeps $Z_{\infty}{ }^{c}$ high and the precious open floc structures intact.

c. Polyvalent Counterions in Coagulation Experiments. The rise of $\varphi_{\delta}^{c}$ with an increase in $z$ can also be important for the coagulation of particles with a small $A$, like polymer latices. Small Hamaker constants correspond to small values of $Z_{\infty}{ }^{c}$. Extreme concentrations of 1-1 electrolyte would be necessary to make $Z_{\infty}<Z_{\infty}{ }^{c}$ in such sols. Coagulation with polyvalent ions is more effective again because lowering of $\varphi_{\delta}$ is combined with a rise in $\varphi_{\delta}^{c}$.

The DLVO theory for the rates of slow and rapid coagulation remains valid, as $Z_{\infty}<Z_{\infty}{ }^{c}$ whenever there is coagulation. This part of the theory is not really affected by assumptions of a constant $\sigma(25)$ and a variable $\varphi_{\delta}(3)$, nor by the subtlety that $H=H_{0}+2 \delta$. These three modifications make a difference for small $H$, but coagulation rates are governed by the maximum in the potential energy diagrams near $\kappa H=1$. That the slope of the experimental $\log W$ $\log C$ curves $(19,34)$ is hardly dependent on $z$ is an indication that $Z_{\infty}$ is rather small in coagulating sols.

For small $Z_{\infty}$ it is easily derived that at the critical coagulation concentration $C^{c}$ there is a rather good proportionality between $\kappa$ and $\varphi_{\delta}^{2}$. This is the theoretical equivalent of the empirical rule of Eilers and Korff (33) that a sol becomes unstable when $\zeta^{2} K^{-1}$ is below some definite value. But for small $Z_{\infty}$ the traditional derivation of the Schulze-Hardy rule does not hold anymore. It was based on the reasoning that according: to the DLVO theory $C^{c} z^{6}$ is proportional with $\tanh ^{4}\left(Z_{\infty} / 4\right)$ and that this would be constant for large $Z_{\infty}$.

It has been pointed out $(33,38)$ that the rule of Eilers and Korff and the Schulze Hardy rule are compatible if $\varphi_{\delta}$ at $C^{c}$ decreases when $z$ increases, and there seems to be some electrokinetic evidence for this. It appears that the lowering of the maxima in the potential energy curves is not only caused by the compression of the double layer but that it is also, and sometimes critically, affected by the change in $Z_{\infty}$ when inert electrolyte is added.

d. Aging, the Irreversibitity of Flocs. It must not be concluded from potential energy diagrams like Figs. 3 and 4 that all flocs from all electrocratic colloids can be repeptized. These diagrams merely show that the aggregation of two particles can be reverted if nothing changes after the collision of the particles until they separate again. The own time scale of the diagrams is that of a Brownian collision.

The temperature-dependent increase in the irreversibility of flocs (2) indicates that rate processes with quite different time scales are at work in a floc and change its properties. Two of these processes are easily recognized: the sintering of particles and the loss of double layer charge from the particle surfaces. The sintering of aggregated particles is analogous to Ostwald ripening. By growing together the particles diminish their interfacial free energy.

The charge $\sigma$ remained constant during a Brownian collision. As a result $\sigma$ exceeds its equilibrium value in a newly formed aggregate. A discharging current will eventually restore the equilibrium, but this is a 
slow and complex process in the tortuous floc structure.

The ultimate result of these sintering and discharging processes combined is predicted by equilibrium thermodynamics: the particle surfaces come into contact at $H=\Delta$. Where they touch they are uncharged and they have the Nernst equilibrium potential $\varphi^{0}$. This situation, where the surface of the original particles has in fact disappeared, was considered as the end of aggregation in the original DLVO theory. It may be reached, but that takes more time than a Brownian collision. If we take so much time in an experiment we shall end up with irrepeptizaable flocs.

In a few special systems it is to be expected that the aging of flocs is all but nonexistent. In a polymer latex the charged end groups are chemically bound and cannot leave the particles. In clay suspensions the particle charge is due-at least partly-to the isomorphous replacement of lattice ions. Such particles cannot lose their charge. There is no reason for the sintering of clay particles either. Each particle is an essentially two-dimensional platelet. If one particle grows at the expense of another there is no change in the surface free energy of the system. These material properties of the particles make us expect that the aging of aggregates will be a slow process in a clay. In such a colloid a floc can remain unchanged and repeptizable for a long time after its formation.

\section{SUMMARY AND CONCLUSIONS}

Versions of the DLVO theory which were intended to describe electrocratic colloids on the verge of coagulation cannot account for such phenomena as repeptization, because their chosen boundary conditions do not apply under the circumstances which bring about repeptization. We proposed three modifications: $\sigma$ remains constant during a Brownian collision; $\varphi_{\delta}$ depends on the electrolyte concentration; and $H=H_{0}$ $+2 \delta$.
In this version of the theory there is a critical value $Z_{\infty}{ }^{c}$ for the double layer parameter $Z_{\infty}=z e \varphi_{\delta} / k T$. For $Z_{\infty}>Z_{\infty}{ }^{c}$ a colloid must be stable and it can coagulate if $Z_{\infty}<$ $Z_{\infty}{ }^{c}$. The behavior of a colloidal dispersion under changes in the salt concentration $C$ or in the counterion valency $z$ is determined to a large extent by variations of $Z_{\infty}$ relative to $Z_{\infty}{ }^{c}$.

It is implicit throughout this version of the theory that the time scale of an experiment is more important for colloid chemical observations than it has often been assumed. In experiments on coagulation and on repeptization $(3,4,11)$ fairly arbitrary time scales have been in use for half a century. The ratio between the time scale of such an experiment and that of the collision of particles or the aging of flocs has to be taken into account in a theoretical interpretation of the results.

\section{APPENDIX}

CALCULATION OF $V_{R^{\sigma}}$ AT CONSTANT $\sigma$ (8).

a. F'lat Double Layers. We shall assume that a constant charge density $\sigma$ of the double layer implies a constant $\sigma$ of the diffuse layer. The charge in a Stern layer can remain constant, either because there is no specific adsorption at all, or because this charge-like the particle charge-does not change fast enough.

The Gouy Chapman theory for a flat diffuse layer gives

$$
\sigma=\left[\frac{n \epsilon l T}{2 \pi}(2 \cosh Z-2)\right]^{1 / 2},
$$

where $\sigma$ is the charge density per unit area of surface; $n$ is the electrolyte concentration in molecules per unit volume, $\epsilon$ is the dielectric constant, $k$ is Boltzmann's constant, and $T$ is the temperature. $Z=z e \varphi_{\delta} / k T$, with $z$ the ionic valency, $e$ the elementary charge, and $\varphi_{\delta}$ the potential in the Outer Helmholtz Plane (O.H.P.).

For one of two interacting double layers with a distance $H$ between the two O.H.P. 


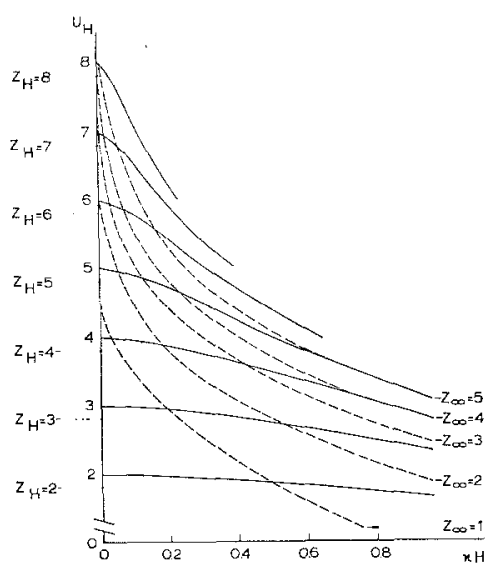

FIG. 5. Dotted: $Z_{H}$ as a function of interparticle distance $(\kappa H)$ for given values of $Z_{\infty}$ (constant $\sigma)$. Drawn: $U_{H}$ as a function of $\kappa H$ for given values of $Z_{H}$ (constant $\varphi_{0}$ ). (see text).

$$
\sigma=\left[\frac { n \epsilon k T } { 2 \pi } \left(2 \cosh Z_{H}\right.\right.
$$

$$
\left.\left.-2 \cosh U_{H}\right)\right]^{1 / 2}
$$

$U_{H}=z e \varphi_{d} / k t$.

where $\varphi_{d}$ is the potential halfway between the two O.H.P.

$\sigma$ is constant, independent of $I I$; therefore

$$
\cosh Z_{H}-\cosh U_{H}=L \text {, }
$$

where $L$ is constant.

Equation [7] is valid for all $H$, so that for a single particle $(H \rightarrow \infty)$

$$
L=\cosh Z_{\infty}-1 \text {. }
$$

The parameter $Z_{\infty}$ is accessible by independent experimentation; e.g., by equating $\varphi_{\delta}=\zeta$ and measuring the electrokinetic $\zeta$-potential, or from measurements of the charge and the capacities of a double layer at an electrode, or by model theories.

From $Z_{\infty}$ one obtains $L$ in Eq. [8], and with $L$ Eq. [7] generates all the pairs $Z_{H}$, $U_{H}$ for $\sigma$ constant and $Z_{H}>Z_{\infty}$. A calculation of ${ }_{\kappa} H$ for arbitrary combinations $Z_{H}$, $U_{H}$ is found in (19). This table together with the pairs $Z_{H}, U_{H}$ from Eq. [7] gives $Z_{H}$ as a function of ${ } H$ for a constant charge (Fig. 5). An analogous result was obtained by van Olphen for larger values of ${ }_{k} H(12)$.

The potential energy $V_{R}{ }^{\sigma}$ of a double layer system consisting of two interacting flat double layers at distance $H$ and with constant $\sigma$ is

$$
V_{R}^{\sigma}=2\left(F_{H}-F_{\infty}\right)
$$

where $F$ is half the electrical part of the Helmholtz free energy of the double layer system. We need only consider the electrical free energy of the diffuse layers, since the electrical free energy of the Stern layer at constant $\sigma$ is independent of $H$. As in reference 19 we put:

$$
F=\int_{0}^{\sigma} \varphi_{\delta} \mathrm{d} \sigma=\sigma \varphi_{\delta}-\int_{0}^{\varphi_{\delta}} \sigma \mathrm{d} \varphi .
$$

Therefore:

$$
\begin{aligned}
V_{R}{ }^{\sigma}=2 & {\left[\left(\sigma \varphi_{\delta}\right)_{H}\right.} \\
& -\left(\int_{0}^{\varphi_{\delta}} \sigma \mathrm{d} \varphi\right)_{H}-\left(\sigma \varphi_{\delta}\right)_{\infty} \\
& \left.+\left(\int_{0}^{\varphi_{\delta}} \sigma \mathrm{d} \varphi\right)_{\infty}\right] .
\end{aligned}
$$

The four terms of this equation can be obtained separately. From the theory for a single flat layer it follows that

$$
\left(\sigma \varphi_{\delta}\right)_{\infty}=\frac{4 n k T}{\kappa} Z_{\infty} \sinh \frac{Z_{\infty}}{2}
$$

and

$$
\begin{aligned}
\left(\int_{0}^{\varphi_{\delta}} \sigma \mathrm{d} \varphi\right)_{\infty}= & \frac{2 n k T}{\kappa} \\
& \cdot\left(4 \cosh \frac{Z_{\infty}}{2}-4\right) .
\end{aligned}
$$

$\sigma$ is constant; therefore

$$
\left(\sigma \varphi_{\delta}\right)_{H}=\left(\sigma \varphi_{\delta}\right)_{\infty} \cdot \frac{Z_{H}}{Z_{\infty}} .
$$

The fourth term can be related to the potential energy $V_{R}{ }^{\psi}$ of two double layers which come together while their $Z$ remains 
constant and equal to $Z_{H} \cdot V_{R}^{\psi}$ is available in extensive numerical tables (16). In reference 19 it is shown that

$$
\begin{gathered}
\left(\int_{0}^{\varphi_{\delta}} \sigma \mathrm{d} \varphi\right)_{H}=-F_{H}{ }^{\psi}, \\
F_{H}^{\psi}=1 / 2 V_{R}{ }^{\psi}+F_{\infty}{ }^{\psi},
\end{gathered}
$$

where $F_{H}{ }^{*}$ is half the free energy of a double layer system with distance $H$ and constant potential $\psi$, for $\psi=Z_{H}(k T / z e)$. According to Eq. [13].

$$
F_{\infty}^{\psi}=-\frac{2 n k T}{\kappa}\left(4 \cosh \frac{Z_{H}}{2}-4\right) .
$$

The combination of Eqs. [12]-[17] gives

$$
\begin{aligned}
V_{R}^{\sigma}=V_{R}^{\psi} & +\frac{8 n k T}{\kappa} \\
\cdot & {\left[\left(Z_{H}-Z_{\infty}\right) \sinh \frac{Z_{\infty}}{2}\right.} \\
& \left.-2\left(\cosh \frac{Z_{H}}{2}-\cosh \frac{Z_{\infty}}{2}\right)\right] .
\end{aligned}
$$

which is Eq. [1] for the interaction energy of two flat double layers with a constant charge. The useful function $f($ U.Z $)=$ $z^{2} \kappa^{-1} V_{R}$ is shown in Fig. 6, both for a constant $\sigma$ and a constant $\varphi_{\delta}$.

b. Spherical Double Layers. The interaction between curved double layers is of more practical importance than that between flat plates, because colloidal particles are seldom flat plates, and even these will only rarely lie face to face in a floc. The repulsion energy of spherical double layers can be calculated from Eq. [1] with Derjaguin's approximation (27) provided that the radius $a$ of the particles is much larger than the Debye length $1 / \kappa$. This is nearly always the case, especially since the electrolyte concentration is never extremely low in problems of colloid stability. Derjaguin's calculation is independent of assumptions about constant $\sigma$ or $\varphi$. It results in a formula

$$
G_{\kappa H_{0}}=2 \pi \int_{H_{0}}^{\infty} f(\mathrm{U}, \mathrm{z}) \mathrm{d} \frac{\kappa H_{0}}{2}
$$

where $G\left({ }_{{ }_{k H}}\right)=z^{2} a^{-1} V_{R}$ for spherical par-

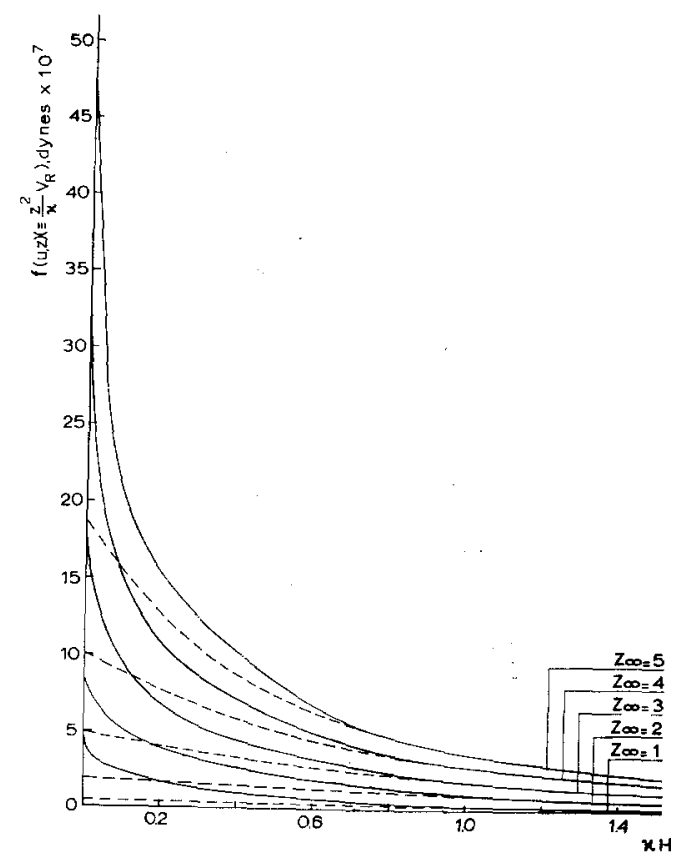

FIg. 6. Repulsion between flat double layers, $f($ v.z. $)=z^{2} \kappa^{-1} V_{R}$ Drawn: constant $\sigma$, dotted: constant $\varphi_{\delta}$.

ticles, and $H_{0}$ is the shortest distance between the two O.H.P. Numerical values of $G_{k H_{0}}^{\varphi}$ for the interaction of two spheres with a constant $\varphi$ have been calculated by Verwey and Overbeek (19). $G_{k H_{0}}^{\sigma}$ for a constant $\sigma$ is obtained by numerical integration of Eq. [1]. Numerical tables will be published shortly (39).

If we rewrite [18] as

$$
\begin{aligned}
G_{\kappa H_{0}} & =2 \pi\left[\int_{H_{0}}^{\infty} f^{\varphi}(\mathrm{v}, \mathrm{z}) \mathrm{d} \frac{\kappa H_{0}}{2}\right. \\
+ & \left.\int_{H_{0}}^{\infty}\left(f^{\sigma}(\mathrm{v}, \mathrm{z})-f^{\varphi}(\mathrm{v}, \mathrm{z})\right) \mathrm{d} \frac{\kappa H_{0}}{2}\right]
\end{aligned}
$$

we obtain Fig. 1 and Eq. [2].

\section{REFERENCES}

1. Frens, G., and Overbeek, J. Th. G., J. Colloid Interface Sci. (submitted).

2. KRUX'T, H. R., AND KLompÉ, M. A. M., Kolloid Beih. 54, 507, (1943).

3. TEZAK, B., Z. Phys. Chem. A 175, 284, B 32 46,52 (1936).

4. Freundlich, H., "Kapillarchemie II", Leipzig (1932). 
5. Gillespie, T., J. Colloid Sci 15, 313 (1960).

6. Pfordten, O. v.d., Ber. D.Ch. Ges. 20, 1458 (1887).

7. Oden, S., Z. Phys. Chem. 78, 682 (1912).

8. Frens. G., Thesis, Utrecht, (1968).

9. KuzeL, H., D. R. P. K1 12 g Nr. A 2572.06.

10. Frens, G., And Overbeer, J. Th. G., Kolloid $Z$. 233, 922 (1969).

11. Rohrsetzer, S., Ann. Un. Sc. Bud. Rol. Eotvos Nom. S. Chim. 7, 77, (1965.)

12. OLphes, H. v., "An Introduction to Clay Colloid Chemistry." New York, 1963.

13. Mackor, E. L., Quoted in ref. (20).

14. TezaK, B., Discussions Faraday Soc. 18, 194 (1954).

15. Stigter, D., quoted in ref. (20).

16. Glasstone, S., Laiduer, K. J., and Eyring, H., "The Theory of Rate Processes." New York, (1941).

17. Fuchs, N., Z. Phys. 89, 736, (1934).

18. Deryagin, B. V., and Landau, L., Acta physicochim. 14, 633, (1941).

19. Verwex, E. J. W., and Overbeer, J. Th. G., "Theory of the Stability of Lyophobic Colloids." Amsterdam, 1948.

20. KruYt, H. R., "Colloid Science," Vol. I, Amsterdam. (1952).

21. Voropaeva, T. N., Deryagin, B. V., AND Kabanov, B , Koll.Zh.24, 396 (1962).

22. Usur, S., Yamasaki, T., AND ShImolizaka, J., J. Phys. Chem. 71, 3195 (1967).
23. Overbeek, J.Th.G., J. Phys. Chem. 64, 1178, (1960).

24. Frens, G., Enged, D. J. C., And Overbeek, J.Th.G., Trans. Faraday Soc. 63, 418 (1967).

25. Jones, J. E., And Levine, S., $J$. Colloid Interface Sci. 30, 241, (1969).

26. DevereuX, O. F., AND DE Bruyn, P. L., "Interactions of Plane-Parallel Double layers." Cambridge, Massachusetts, 1963.

27. Deryaguin, B. V., Kolloid $Z$. 69, 155, (1934).

28. HAMAKER, H. C., Physica 4, 1058, (1937).

29. Grahame, D. C., Chem. Rev. 41, 441, (1947).

30. Reerink, H. Thesis, Utrecht, Netherlands, 1952.

31. Levine, S., Mingins, J., And Beli, G. M., $J$. Electronal. Chem. 13, 280, (1967).

32. Wiersema, P. H., and Loeb A. L., and OverBEEK, J.Th.G., Colloid Interface Sci. 22, 78 (1966).

33. Erluens, H., AND KonfF, J., Trans. Faraday Soc. 36, 299, (1940).

34. Watillon, A., and Joseph-Petit, A. M., Discussions Faraday Soc.42, 143 (1966).

35. Krupp, H., Adv. Coll. Int. Sc. 1, 111, (1976).

36. Mrsels, K. J., "Introduction to Colloid Chemistry." New York, 1959 (1959).

37. ref. (19), fig. 40.

38. Barbot, V. M., Koll.Zh. 25, 385 (1963).

39. Honig, E. P., Private communication. 